médecine/sciences $1993 ; 9: 50-8$

\title{
De la musique au cerveau, par l'intermédiaire de Maurice Ravel
}

Justine Sergent

ADRESSE ET TIRÉS À PART

J. Sergent : Laboratoire de neurosciences cognitives, Institut neurologique de Montréal, Université Mc Gill, 3801 rue Univer-

La compréhension de l'organisation fonctionnelle des structures cérébrales sous-jacentes aux processus réceptifs et expressifs de la musique, malgré les difficultés à laquelle elle se heurte, est un objet légitime de la recherche scientifique. L'étude de cas de musiciens, tel que Maurice Ravel, ayant été victimes d'accidents cérébraux offre la possibilité de découvrir certaines caractéristiques des relations entre la musique et le cerveau, permet d'identifier les questions essentielles posées par ces relations et de formuler des hypothèses. En utilisant les nouvelles techniques d'imagerie cérébrale, comme l'étude tomographique par émission de positons et l'examen cérébral par résonance magnétique, ces hypothèses peuvent être soumises à vérification. Les résultats de ces recherches montrent que la réalisation des fonctions musicales par le cerveau engage un large réseau neuronal qui englobe les quatres lobes cérébraux et le cervelet et met en jeu des structures cérébrales adjacentes mais distinctes de celles qui sous-tendent le langage verbal. Il existe donc une relative indépendance fonctionnelle et structurale du langage verbal et du langage musical, qui explique l'existence de dissociations entre les troubles de ces deux fonctions à la suite d'une lésion cérébrale.

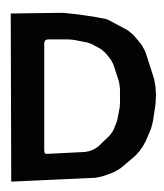

e toutes les facultés cognitives humaines, peu paraissent aussi hermétiques à l'investigation scientifique que les capacitćs musicales. Comprendre les processus psychologiques inhérents à l'audition ou à l'exécution musicale et identifier les substrats neurobiologiques qui les sous-tendent se heurtent à des difficultés multiples auxquelies l'étude des autres fonctions cognitives ne se trouve guère exposée. Ainsi, l'écoute de la musique constitue une expérience personnelle diffi- cilement communicable et elle est influencée par des facteurs tels que l'intérêt, l'éducation, la culture, la personnalité, de sorte qu'un morceau de musique qui suscite une émotion intense chez certains individus peut laisser d'autres complètement indifférents. L'expression musicale, quant à elle, relève du domaine artistique et se trouve être le fait d'une minorité ; de plus, elle requiert l'acquisition de techniques et de pratiques que chaque musicien développe et perfectionne à sa façon. Autant l'écoute que l'expression musicales sont donc 
peu propices à l'approche expérimentale scientifique qui, traditionnellement, porte sur des données quantifiables et repose sur l'étude de groupes homogènes dans le but d'aboutir à des généralisations à partir d'un échantillon représentatif.

Pourtant, la compréhension des relations entre la musique et le cerveau, c'est-à-dire de l'organisation fonctionnelle des stuctures cérébrales sousjacentes aux processus réceptifs de la musique, est un but légitime de la recherche scientifique. D'une part, la musique peut être considérée comme un langage, et le langage musical possède une grammaire, une syntaxe et des règles d'exécution qui, bien que variables selon les époques et les régions, n'en sont pas moins particulières à la musique et donc distinctes de celles qui caractérisent le langage verbal [1-3]. En ce sens, la réalisation des fonctions musicales engage des processus mentaux qui leur sont propres et s'appuie donc sur des structures cérébrales dotées des propriétés permettant la mise en jeu de ces processus spécifiques du domaine musical. De fait, les recherches portant sur les patients ayant des lésions cérébrales ont montré que la perte du langage verbal (aphasie*) n'était pas nécessairement accompagnée d'une perte du langage musical (amusie*) [4, 5]. Ainsi, l'existence d'aphasies sans amusie et d'amusies sans aphasie met en évidence une double dissociation suggérant autant l'autonomie fonctionnelle des processus mentaux inhérents au langage verbal et musical que l'indépendance structurale de leurs substrats neurobiologiques. Néanmoins, les deux déficits sont fréquemment associés, indiquant, soit que ces deux fonctions font appel à certaines opérations communes, soit que leurs substrats neurobiologiques respectifs sont distincts mais adjacents, et peuvent donc être conjointement affectés par des lésions qui respectent rarement les territoires fonctionnels neuronaux [6]. Par ailleurs, la pratique de la musique est un exemple typique d'opérations mentales multimodales, puisqu'elle fait appel à la modalité visuelle par le biais de la notation musicale, à la modalité auditive au niveau de la

\footnotetext{
- Voir glossaire, p. 51.

$m / s n^{\circ} 1$ vol. 9, janvier 93
}

\section{GLOSSAIRE}

Agnosie : trouble de la reconnaissance ou de la signification des informations sensitives.

Agraphie : désorganisation de l'expression écrite due à des lésions cérébrales, à l'exclusion des troubles de la réalisation graphique liés à une atteinte de la voie motrice principale des systèmes de contrôle du mouvement (cérébelleux et striés essentiellement).

Alexie : trouble acquis correspondant à l'incapacité de comprendre les mots écrits ou imprimés, engendré par une lésion cérébrale focalisée.

Amusie : perte du sens musical, sans altération de l'acuité auditive, rendant le sujet incapable de reconnaître une musique.

Aphasie : altération du langage oral et écrit causées par des lésions de l'hémisphère majeur affectant le lobe temporal ou la 3e circonvolution frontale, et sans rapport avec des phénomènes paralytiques ni des lésions cérébelleuses ou extrapyramidales, ni une atteinte sensorielle, ni un état démentiel.

Aphasie de Wernicke : aphasie où prédominent les troubles de la compréhension (surdité verbale et alexie), l'expression étant également perturbée de façon souvent importante (paraphrasie, jargonophasie).

Apraxie : trouble acquis de l'exécution du mouvement volontaire, indépendant des phénomènes, parétiques ou ataxiques, d'atteinte striée ou cérébelleuse et d'un déficit intellectuel.

Gyrus angulaire : partie postérieure de la circonvolution pariétale inférieure qui se continue par les deux premières circonvolutions temporales.

Gyrus supramarginal : segment antérieur de la circonvolution pariétale inférieure correspondant à l'extrémité postérieure de la scissure de Sylvius.

Prosopagnosie : agnosie particulière au cours de laquelle le sujet est incapable de reconnaître les visages, même familiers, qui sont en sa présence ou représentés sur une photographie. réception des mélodies, des harmonies et des rythmes dont la combinaison définit unc œuvre musicale, à la modalité motrice en ce qui touche l'exécution qui requiert virtuosité et coordination d'ensembles musculaires, sans oublier les aspects cognitifs et émotionnels nécessaires à l'interprétation et à l'appréciation de la musique. En ce sens, une étude neurocognitive des fonctions musicales déborde le simple cadre de la musique et offre la possibilité d'examiner les mécanismes, et découvrir les principes, par lesquels le cerveau organise ses ressources lorsqu'il lui faut coordonner une variété d'opérations mentales.

Parmi les approches expérimentales disponibles, deux se révèlent particulièrement appropriées à ces objectifs. L'une est l'étude de cas de musiciens ayant été victimes d'accidents cérébraux, qui vise ainsi à établir des corrélations entre, d'une part, les fonctions affectées et préservées dans le domaine musical ou autre et, d'autre part, la nature et la localisation cérébrale de la perturbation. La seconde approche s'appuie sur les développements récents de l'imagerie cérébrale fondée sur la tomographie par émission de positons (TEP) [7, 8]. Outre une visualisation de la neuro-anatomie fonctionnelle de processus mentaux spécifiques, cette technique permet d'examiner les relations entre le cerveau et les fonctions cognitives en sélectionnant des sujets sains et normaux qui présentent des caractéristiquent homogènes et appropriées à l'objet d'étude. Cet article décrit l'application de ces deux approches au problème des bases neurobiologiques de certaines capacités musicales.

\section{Musiciens victimes de troubles cérébraux}

Parmi les grands compositeurs de ce siècle, certains eurent à endurer les effets de lésions cérébrales dont les manifestations varièrent selon les cas. Ainsi le compositeur russe et directeur du conservatoire de Moscou, Vissarion Iakovlev Chebaline (1902-1963), fut victime de deux hémorragies cérébrales (1953 et 1959) dont la seconde produisit une sévère 


\section{RÉFÉRENCES}

1. Deliège C. Les fondements de la musique tonale. Paris : Lattès, 1984.

2. Sloboda JA. L'esprit musicien. Liège : Mardaga, 1985.

3. Lerdhal F, Jackendorff R. A generative theory of tonal music. Cambridge MA : MIT Press, 1983

4. Benton AL. The amusias. In : Critchley M, Henson RA, eds. Music and the Brain. London : Heinemann, 1966 : 378-97.

5. Marin O. Neurological aspects of music perception and performance. In : Deutsch D, ed. The Psychology of Music. New York: Academic Press, 1982 : 453-77.

6. Brust JCM. Music and language. Musical alexia and agraphia. Brain $1980 ; 103$ : 367-92.

7 Changeux JP. Les neurones de la raison. La Recherche 1992 ; 244 : 704-13.

8. Sergent J. Neuro-anatomie fonctionnelle de l'identification des visages : une étude tomographique par émission de positons. Rev Neuropsychol 1991; 1 : 119-56.

9. Luria AR, Tsvetkova LS, Futer DS. Aphasia in a composer. J Neurol Sci 1965 ; 2 : 288-92.

10. Signoret JL, Van Ecckhout P, Poncet M, Castaigne P. Aphasic sans amusic chez un organistc aveugle. Alexie-agraphic verbale alcxic-agraphie musicalc en braille. Rev Neurol 1987; 143 : 172-81.

11. Henson RA. Maurice Ravel's illness : a tragedy of lost creativity. $\mathrm{Br}$ Med J 1988 : $296: 1585-8$

12. Carp L. George Gershwin, illustrious Amcrican composer : his fatal glioblastoma. Am J Surg Pathol 1977 ; 3 : 473-8.

13. Alajouanine T. Aphasia and artistic realization. Brain 1948 : 71 ; 229-41. (traduction française : Réalisation artistique ct aphasic. In : Alajouaninc $\mathrm{T}$, ed. L'Aphasie et Langage Pathologique. Paris : Baillère 1968 : 275-300).

14. Jankélévitch V. Ravel. Paris : Scuil, 1956.

15. Jourdan-Morhange $H$. Ravel et nous. Genève: Éditions du milieu du monde, 1945.

16. Long M. Au piano avec Maurice Ravel. Paris: Gérard Billaudot Éditeur, 1971

17. Marnat M. Maurice Ravel. Paris : Fayard, 1986.

18. Roland-Manuel A. Ravel. Paris : Galli- aphasie de Wernicke* ; il n'en continua pas moins de composer sans diminution apparente - selon son collègue Chostakovitch - de ses capacités musicales [9]. Il en fut de même de l'organiste et compositeur français Jean Langlais (1907-1991) qui devint aphasique à la suite d'un accident vasculaire cérébral et dont les troubles de lecture et d'écriture se limitaient au langage verbal sans affecter la langage musical [10]. Le compositeur anglais Benjamin Britten (1913-1976) fut victime d'une embolie cérébrale qui n'eut aucun effet significatif ou durable sur ses capacités verbales ou musicales mais réduisirent la mobilité de son bras droit, produisant ainsi une gêne pour l'écriture [11]. Plus tragique fut la tumeur cérébrale (glioblastome), dans le lobe temporal droit, dont souffrit le compositeur américain George Gershwin (1898-1937) [12]. La progression de sa maladie fut si rapide que, mis à part de brèves absences, aucun signe de déficit des fonctions cognitives ne put être noté, même durant les dernières semaines précédant sa mort à l'âge de 38 ans. Aucun de ces cas ne permet d'émettre des hypothèses sur les relations entre le cerveau et la musique, si ce n'est pour suggérer que les structures cérébrales dont la destruction provoque une aphasie ne sont pas indispensables aux processus de composition musicale, ce qui ne signifie pas que ces régions ne participent pas normalement à ces processus ou ne soient pas impliquées dans d'autres aspects des fonctions musicales.

Le cas la plus dramatique est sans aucun doute celui de Maurice Ravel (1875-1937) dont les quatre dernières années de la vie se déroulèrent dans un état d'incapacité à composer et à jouer de la musique alors qu'il avait conservé ses facultés d'écoute et d'interprétation musicales, la mémoire de ses propre œuvres et ses connaissances musicologiques. Cette maladie tragique n'en est pas moins riche d'informations et mérite donc une attention particulière pour la compréhension des relations entre le cerveau et la musique.

\section{La maladie de Maurice Ravel}

Il existe plusieurs sources d'informa- tions concernant les dernières années de la vie de Ravel et sa maladie. La plus utile est l'article du neurologue Th. Alajouanine décrivant les résultats de ses examens de Maurice Ravel s'étalant sur une période de deux ans [13]. Même si ce rapport fait état de certaines contradictions et n'aborde ni la question de la sévérité des déficits de Ravel, ni celle de la chronologie des symptômes et de l'évolution de la maladie, il n'en constitue pas moins un document unique et essentiel. Des informations supplémentaires peuvent être glanées dans de nombreux ouvrages écrits par des amis de Ravel et des musicologues [14-18]. Cependant, certaines incertitudes demeurent quant à l'exactitude de toutes les informations contenues dans ces ouvrages puisque plusieurs auteurs rendent compte différemment d'événements ou de faits identiques.

Il semble que l'on puisse affirmer que les premiers symptômes indiscutables de troubles cérébraux chez Maurice Ravel furent de nature agraphique*, alexique* et apraxique* et peuvent être datés de l'année 1933. Áinsi, le chef d'orchestre Manuel Rosenthal $[19,20]$, un élève et ami de Ravel, décrit un incident au cours duquel il remarqua, sur la partition d'une composition à laquelle Ravel était en train de travailler (Don Quichotte à Dulcinée, trois chansons accompagnées d'un orchestre et composées fin 1932 -début 1933), une faute d'orthographe au mot "percussion ". Lorsque Rosenthal en fit la remarque à Ravel, celui-ci fut incapable de réaliser son erreur, lui qui attachait une grande importance à la qualité et à la précision dans tous les aspects de son travail. Ses lettres écrites à cette époque sont en fait parsemées de fautes d'orthographe qu'on ne trouve pas dans ses lettres antérieures [21]. Particulièrement révélatrice est l'opinion du musicologue René Chalupt [22] selon laquelle la partition de Don Quichotte à Dulcinée aurait été écrite " par une main amie ", en raison des différences d'écriture entre cette partition et les précédentes. Or, selon Manuel Rosenthal (communication, personnelle, mars 1992-mai 1992), Ravel a composé ces chansons et en

* Voir glossaire, p. 51. 


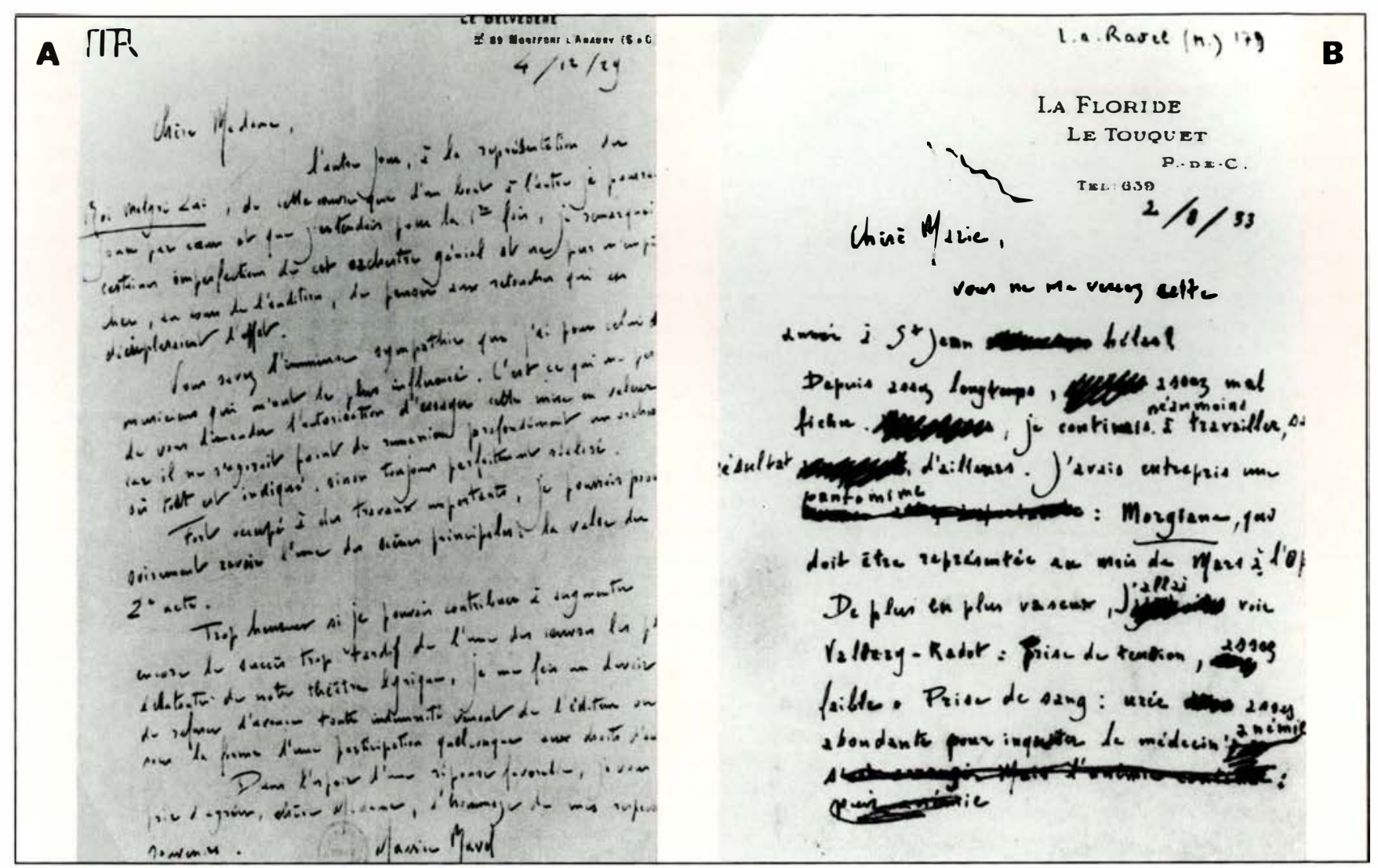

Figure 1. Échantillons de l'écriture de Maurice Ravel. (A) lettre écrite en 1929, à une période où aucun signe de troubles neurologiques ne s'était encore manifesté; (B) lettre écrite en août 1933, alors que les premiers signes d'agraphie et d'apraxie idéomotrice s'étaient manifestés quelques mois auparavant. La destinaire de la lettre était une amie d'enfance de Maurice Ravel; deux mois plus tôt, celui-ci, voulant lui montrer comment faire des ricochets sur l'eau, lança la pierre dans son visage, une maladresse probablement due à l'apraxie (reproduction autorisée par Mme Taverne).

a écrit l'orchestration sans aucune aide, ce qui suggère que les différences graphiques observées sont en fait les premières manifestations de ses troubles neurologiques. Il semble donc qu'à une période où Ravel était encore en possession de ses capacités de composition musicale, il était déjà sujet à des difficultés reliées à l'écriture. Celles-ci iront en s'aggravant, comme en témoigne la lettre d'août 1933 (figure 1b), et, vers la fin de cette même année, il sera incapable de signer son nom et aura perdu la capacité de lire [23]. En juin 1933, alors qu'il se trouve en vacances à Saint-Jean-de-Luz, Ravel se rendit compte qu'il ne pouvait plus effectuer les mouvements de la nage, lui qui était un excellent nageur. Ces premiers signes d'apraxie idéomotrice, déjà précédés d'un ralentissement $m / s n^{\circ} 1$ vol. 9, janvier 93 dans l'exécution de gestes courants, en préfigureront bien d'autres puisqu'il éprouvera par la suite beaucoup de difficultés à accomplir des gestes simples tels que tourner la clé dans une serrure ou même tenir les couverts convenablement dans sa main [17]. C'est aussi à cette époque que les premiers signes de troubles du langage se manifestèrent, par une difficulté à retrouver les noms propres puis les noms courants. Toutefois, bien que ce déficit reflète une forme d'aphasie (de Wernicke, selon Alajouanine [13]), il n'a jamais atteint un degré de sévérité comparable à ses troubles de lecture et d'écriture et Ravel fut, tout au long de sa maladie, capable de s'exprimer intelligemment et de comprendre ce que les autres lui disaient (M. Rosenthal, communication personnelle).
Il est difficile de déterminer avec exactitude quand Ravel s'aperçut pour la première fois d'un déficit directement relié à la musique. En septembre 1933, le journal Excelsior publia un entretien avec Ravel dans lequel il décrivait en détail sa conception de l'opéra Jeanne D'Arc qu'il projetait de composer. Compte tenu de sa grande honnêteté intellectuelle et intégrité morale (" une sincérité désarmante " selon Gabriel Fauré), il est peu probable que Ravel se fût livré à de telle confidences s'il s'était su incapable de composer. Toutefois, autant dans cet entretien que dans une lettre d'août 1933 (figure 1b), il faisait part de sa fatigue et indiquait que ses efforts pour composer demeuraient vains. Le premier document faisant état d'une prise de conscience par Ravel d'une incapacité à compo- 


\section{RÉFÉRENCES}

19. Rosenthal M. Appelcz-moi au 89 à Montfort. Souvenirs de Manucl Rosenthal. Le Monde de la Musique 1987 ; 103 : 114-118.

20. Saudinos D. Manuel Rosenthal. Une vie. Paris : Mercure de France, 1992.

21. Ravel M. Lettres, écrits et entretiens (préscntés ct annotés par Arbie Orenstcin). Paris : Flammarion, 1989.

22. Chalupt R, Gérar M. Ravel au miroir de ses lettres. Paris : Laffont, 1956.

23. Nichols R. Ravel remembered. New York : W. W. Norton and Compagny, 1988.

24. Hugo V. Trois souvenirs sur Ravel. Revue Musicale 1952 ; 137-146.

25. Corballis MC. The lopsided ape. New York : Oxford University Press, 1991.

26. Myers RH. Ravel. Life and works. Westport, CT : Geenwood Press, 1980.

27. Cytowic RE. Aphasia in Maurice Ravel. Bull Los Angeles Neurol Soc 1976 ; 41 : 109-14.

28. Rentchnik P. Mauricc Ravel. Médecine et Hygiène 1983, 41: 2604-16.

29. Mesulam MM. Slowly progressive aphasia without generalized dementia. Ann Neurol $1982 ; 11$ : 592-8.

30. Hécaen H. La neuropsychologie humaine. Paris : Masson, 1972.

31. Sergent J, Signoret JL. Functional and anatomical decomposition of facc processing : evidence from prosopagnosia and PET study of normal subjects. Phil Trans $R$ Soc Lond B 1992 ; 335 : 55-62.

32. Sergent J, Zuck E, Teraiah, Mac Donald B. Distributed ncural network underlying musical sight-reading and keyboard performance. Science 1992; 257 : 106-9.

33. Sergent J, Zuck E, Lévesque M, Mac Donald B. Positron emission tomography study of letter and object processing : Empirical findings and methodological considerations. Cereb Cortex 1992 ; 2 : 68-80

34. Posner MI, Petersen SE, Fox PT, Raichle ME. Localization of cognitive functions in the brain. Science $1988 ; 240$ 1627-31.

35. Judd T, Gardner H, Geschwind $\mathrm{N}$. Alexia without agraphia in a composer. Brain 1983 ; 106 : 435-57.

36. Souques A, Baruk H. Autopsie d'un cas d'amusie (avec aphasic) chez un professeur de piano. Rev Neurol 1930 ; 37 : 545-56.

- Voir glossaire, p. 51. ser date de novembre 1933. Il s'était alors confié à son amie Valentine Hugo en lui disant: "je ne ferai jamais ma Jeanne D'Arc ; cet opéra est là, dans ma tête, je l'cntends mais je ne l'écrirai jamais. C'est fini, je ne peux plus écrire ma musique " [24]. Bien que Ravel ait fréquemment fait part à ses amis de cette incapacité à transposer soit en sons (par le chant), soit en notes (par l'écriture musicale), soit en gestcs (en jouant au piano) les airs et harmonies qu'il disait " entendre dans sa tête ", Alajouanine [13] ne s'est pas penché directement sur ce déficit central de la maladie de Ravel. Ce qui ressort de l'examen mené par Alajouanine est une dissociation entre les aspects auditif, réceptif et interprétatif de l'expérience musicale, qui sont essentiellement préservés, et les aspects productifs et expressifs, qui ne peuvent plus être réalisés.

Ainsi Ravel ne pouvait plus déchiffrer une partition, copier une partition, jouer des morceaux de musique au piano (à l'exception du début de certaines de ses propres compositions), effectuer une dictée musicale (nommer des notes entendues ou les transcrire sur une portée), composer. Il avait toutefois conservé certaines capacités à écrire sur une portée des notes dont le nom lui était donné, et il pouvait écrire et chanter de mémoire quelques unes de ses compositions, avec fautes et hésitations cependant. De plus, il parvenait à reconnaître une œuvre de musique à partir de la partition (qu'il ne pouvait toutefois pas jouer) et était également capable de jouer les gammes majeures et mineures au piano. Ses capacités perceptives et auditives n'étaient pas affectées, qu'il s'agisse des sons, des mélodies ou des rythmes. Sa mémoire de ses propres compositions était intacte jusque dans les plus petits détails, de sorte qu'il détectait la moindre erreur, autant au niveau des notes qu'à celui des aspects plus qualitatifs reliés aux nuances d'interprétation de ses œuvres. En fait, jusqu'à la fin de sa vie, il sera capable de conseiller les interprètes de ses compositions avec la perspicacité et la précision qui l'ont toujours caractérisé. Ainsi, il fournira en 1937 au pianiste Jacques Février [23] des conseils utiles à l'exécution $\mathrm{du}$ Concerto pour la main gauche et, durant l'été de la même année, il corrigera Francis Poulenc et Madeleine Grey qui s'étaient permis certaines libertés mineures dans l'exécution de mélodies de sa composition [23].

La maladie de Ravel consiste donc en une atteinte sélective de fonctions impliquées dans la transposition d'informations d'une modalité à une autre, c'est-à-dire la traduction d'informations auditives en activités motrices, d'informations visuelles en activités motrices, ou d'informations auditives en leurs représentations visuelles et vice-versa, sans qu'aucune des modalités majeures ne soient en elles-mêmes affectées. En fait, du point de vue purement moteur, Ravel ne démontre pas de déficit et peut même jouer les gammes au piano ; il ne présente aucun trouble de la sphère auditive ; à partir des divers documents portant sur les dernières années de sa vie, il peut aussi être établi que, dans le domaine visuel, il ne souffrait ni de négligence spatiale, ni de difficultés d'orientation ou de perte de mémoire topographique, et il n'avait ni agnosie* d'objet ni prosopagnosie* $^{*}[17,18]$. De plus, les transpositions inter-modalités qui s'avèrent défectueuses portent sur des informations et des activités ayant requis un apprentissage spécifique et, de la même façon que son agraphie et son alexie le rendent fonctionnellement illettré, ses troubles reliés à la lecture, l'écriture et l'exécution musicales le rendent musicalement illettré. Les compétences musicales qui lui restent pourraient ainsi être comparées à celles d'un mélomane ou d'un critique musical hautement averti qui n'aurait plus à sa disposition l'usage des connaissances techniques qui constituent les outils de base d'un compositeur. Cela ne signifie pas que Ravel ait perdu ses connaissances techniques, mais il ne peut plus les utiliser parce que leur réalisation fait appel à une intégration d'informations englobant plusieurs modalités.

\section{Nature de la maladie et localisation cérébrale}

Seules nos connaissances anatomocliniques actuelles peuvent nous permettre de formuler des hypothèses sur 


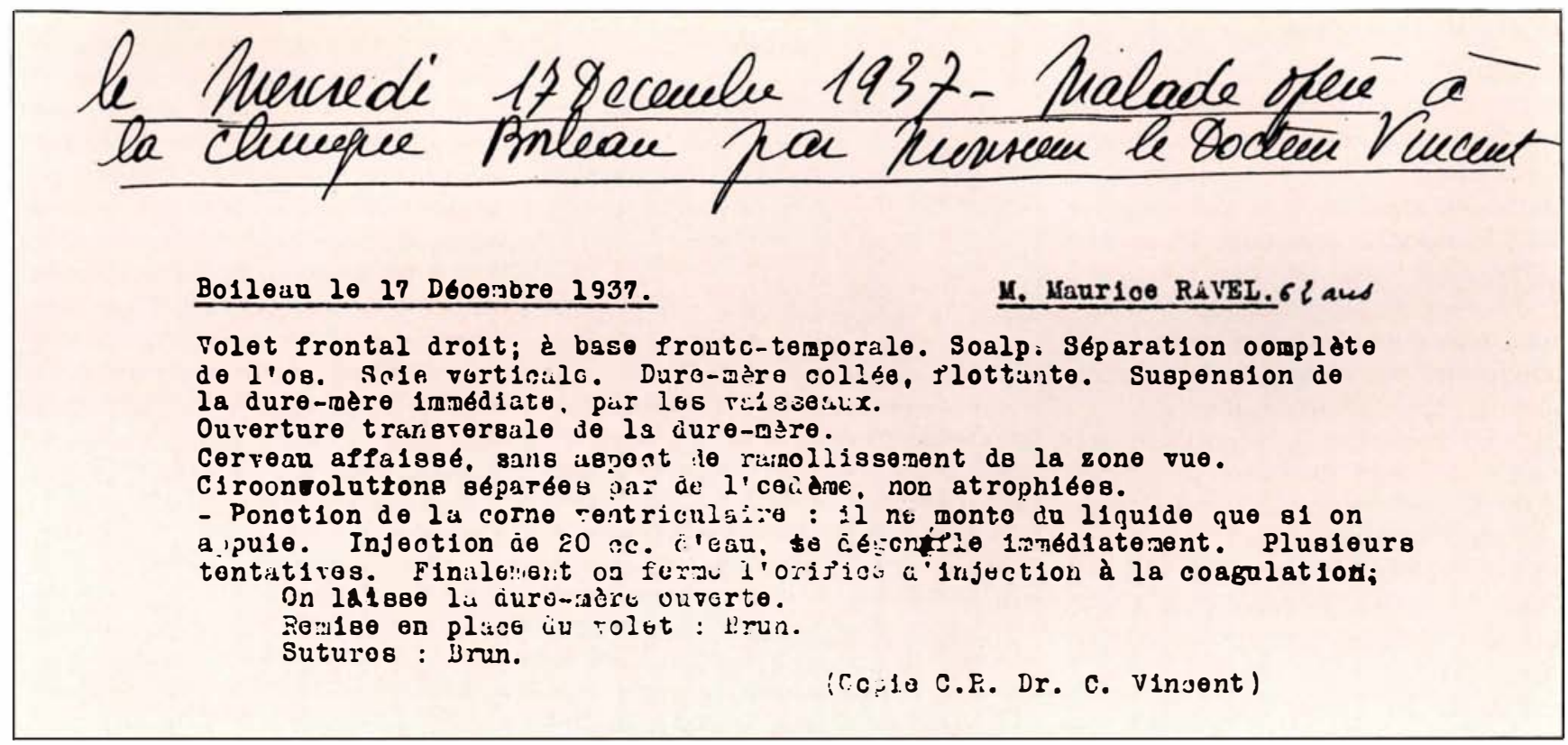

Figure 2. Compte rendu de l'opération chirurgicale pratiquée sur Maurice Ravel par Clovis Vincent à la Clinique Boileau, Paris $16^{\mathrm{m} m e}$, tel que répertorié dans les registres du laboratoire de la Clinique Neurochirurgicale de I'Hôpital de la Pitié à Paris (reproduction autorisée par le professeur Jean Racadot).

les aires cérébrales dont la pertubation fut à l'origine de la maladie de Ravel. Plusieurs auteurs ont avancé des conclusions sans fondement concernant la cause de cette maladie et la localisation de la lésion. Le déficit dc Ravel a ainsi été attribué à un traumatisme crânien [25], à une embolic cérébrale [26], à une hémorragie cérébrale [27], à une lésion du lobe temporal gauche [28], alors qu'il n'existe aucune évidence clinique à ce sujet. Ravel fut victime d'un accident d'automobile en octobre 1932, alors qu'il circulait dans un taxi à Paris, mais il ne reçut ni blessure ni coup à la tête $[17,18]$, mais subit des coupures au visage et un traumatisme thoracique. Il n'en continua pas moins à composer après cet accident puisque, dans unc lettre datée du 6 avril 1933, il écrivait : "Je viens de terminer Don Quichotte à Dulcinée et me mets à Morgiane [21] ". Plusieurs faits sont maintenant établis, sur la base du compte rendu opératoire (figure 2) : il existait une dilatation des ventricules, observéc lors d'un examen pré-opératoire, sans qu'il s'agisse d'hydrocéphalie ; l'opération chirurgicale pratiquée par Clovis Vincent le 17 décembre 1937 n'a permis de visualiser que la partie latérale du lobe frontal droit ct de constater : (1)

$\mathrm{m} / \mathrm{s} n^{\circ} \perp$ vol. 9, janvier 93 un affaissement du cerveau, sans ramollissement ni atrophie de la zone vue, et (2) une très faible pression ventriculaire ; la présence d'une tumeur semble écartée, même si ceci ne peut être prouvé, et la lente évolution de la maladie, dont les symptômes changèrent à peine durant les quatres années qui suivirent ses premières manif estations et demeurèrent restreints à quelques facultés cognitives, milite contre un diagnostic de démence de type Pick ou Alzheimer. Henson [11] a émis l'hypothèse d'une dégénérescence focale progrcssive affectant les zones corticales du langage de l'hémisphère gauche, dont quelques cas ont déjà été rapportés [29]. Cependant, cette explication, quoique la plus plausible, ne rend compte ni de l'affaissement du cerveau, présent au moins dans la zone frontale droite, ni de la dilatation et de la faible pression intraventriculaire en l'absence d'atrophie, ni de l'apparente stabilité des symptômes. Il faut donc admettre que la nature de la maladie de Ravel ne peut être que l'objet de spéculations.

En revanche, il semble plus aisé d'identifier les zones cérébrales dont le dysfonctionnement est susceptible d'être à l'origine de ses troubles cognitifs. D'une part, la présence d'aphasic, même légère, d'apraxie idéomotrice, en l'absence de troubles topographique, spatial ct prosopagnosique, indique avec une forte probabilité une atteinte de l'hémisphère gauche $[30,31]$. D'autre part la préscnce conjointe d'apraxie, agraphie et alcxie, avec une aphasic légèrc de typc Wernicke, suggère une perturbation de la région postérieure de cet hémisphère, probablement dans le territoire couvrant la partie supérieure du lobe temporal et lc lobule pariétal inférieur (gyrus angulaire* et gyrus supramarginal*, figure $3 a$ ) [30]. Il n'en demeure pas moins que cette localisation est clle aussi spéculative, d'autant que les auteurs ayant cffectué des recherches portant sur les musiciens victimes de lésion cérébrale ont toujours incité à la prudence lorsqu'il s'est agi de tirer des conclusion à partir de cas uniques $[4,5]$.

\section{Étude tomographique par émission de positons (TEP)}

Les nouvelles techniques d'imagerie cérébrale peuvent donc nous permettre d'examiner et de vérifier les hypothèses émises sur la base des déficits

* Voir glossaire, p. 51. 
dont sont victimes des patients tels que Ravel. De façon plus spécifique, les questions laissées en suspens par lc manque d'informations concernant la maladic de Ravel peuvent scrvir à mettre sur pied un protocolc cxpérimental visant à comparer les zones cérébrales activécs lors de l'exécution de diverses tâches musicales par des musiciens possédant eux-mêmes les connaissances et techniques qui furent affectécs chez Maurice Ravel.

Un tcllc approche a réccmment été utilisée à l'aide dc la mesure, par TEP, du débit sanguin cérébral à la suite de l'injection d'un marqucur radioactif $\left({ }^{15} \mathrm{O}\right)$ dans le but d'examiner les zones du cerveau spécifiquement activées lors de la performance d'unc variété d'ćpreuves faisant appel à certaines des capacités perturbées chez Mauricc Ravel [31]. Ces épreuves furent exécutées par des pianistes professionncls droitiers (huit hommes et deux femmes dont l'âge variait entrc 24 ct 32 ans). De façon à identifier les zones cérébralcs sous-jacentes à ces diverses fonctions musicales, une série de soustractions fut cffectuée visant à isoler, parmi les régions faisant état d'unc augmentation du débit sanguin provoquéc par unc épreuve donnéc, celles dont la participation est spécifiquc à cettc éprcuvc. Ainsi, pour identifier les régions impliquées dans la composante motrice de l'exécution des gammes sur un piano, l'activation cérébrale associée à l'écoute de gammes jouées au piano fut soustraitc de l'activation associée à l'écoutc et à l'cxécution de gammes jouécs au piano par les sujcts. Bien qu'unc telle méthode nc soit pas sans poser des problèmes théoriques et pratiques [32], la justesse de l'identification des zoncs cérébrales impliquées a été validéc à l'aide de comparaisons avcc les donnécs anatomiques de patients souffrant de déficits bien circonscrits [30]. Par ailleurs, une image du ccrvcau de chaque sujet fut obtenue par résonance magnétique dans le but d'identifier, par supcrposition des données physiologiques sur les données anatomiques, les structures cérébrales spécifiquement activées lors de la réalisation de chaque éprcuve [31]. Les résultats ont permis de fairc

\section{Tableau I}

\section{PROTOCOLE EXPÉRIMENTAL}

Liste des épreuves exécutées par dix pianistes professionnels durant la mesure du débit sanguin cérébral dans l'étude tomographique par émission de positons. Chaque épreuve durait une minute, séparée par un intervalle de 12 minutes. Par un processus de soustraction de l'activation associée à la réalisation de chacune des épreuves, il est possible d'identifier les zones cérébrales qui participent aux composantes cognitives différenciant deux épreuves.

\section{Condition contrôle de base} fixation passive de l'écran

\section{Condition contrôle visuo-moteur présentation de points}

et réponse manuelle en fonction de leur localisation

Condition contrôle auditif écoute passive de gammes majeures jouées au piano

\section{Condition contrôle de pratique du piano}

exécution de gammes majeures jouées et entendues par le sujet

Condition expérimentale lecture musicale

lecture silencieuse d'une partition d'un choral de J. S. Bach

Condition expérimentale

lecture et écoute musicale lecture de partition d'un choral de J. S. Bach

et écoute de cette musique

Condition expérimentale - déchiffrage lecture, exécution et écoute d'une partition de J. S. Bach

ressortir l'existence d'un riche réseau neuronal sous-jacent au déchiffragc, à l'exécution et à l'écoute d'œuvres musicales qui s'étend sur les quatre lobes cérébraux ainsi que sur le cervelet. Certains aspects méritent une attention particulièrc.

1. La lecture d'une partition a mis en jeu le territoire occipito-pariétal qui est normalement "silencieux" durant la lecture verbale mais n'a pas engagé les zones cérébrales du cortcx occipital ventral qui participent normalement à la lecture de mots [33]. Cette différence ticnt principalement à ce que la lecture de notes de musique ne requiert pas en priorité une analyse perceptuelle visant à discriminer chaque lettre en fonction de sa formc, mais fait plutôt appel à une analyse, d'une part, de la position des notes sur la portée, d'autre part, de l'intervalle séparant deux notes adjacentes ct directement relié à la différence de hauteur entre ccs deux notes. L'analyse structurale des notes a donc moins d'importance que leur analyse spatiale dans la lecture musicale, et les deux types de lecture mettent en jeu des opérations cognitives distinctes, sous-tendues par des structures corticales différentes. Cettc dissociation des processus sous-jacents aux deux types de lecture, et de leurs substrats ncurobiologiques, peut ainsi expliquer pourquoi ccrtains musiciens devenus "alexiques verbaux" conscrvent leur capacité à lirc et à déchiffrer des partitions musicales [34].

2. La lecture et l'écoute d'unc œuvre musicale ont conjointement provoqué l'activation du cortex parićtal des deux hémisphères. Le fait que ni la lecture seulc, ni l'écoutc seule, n'ont entraîné une activation de cette zone suggère quc ccttc structure participe à la mise en correspondance des informations visuelles et auditives et contribuent donc à la "traduction" de notes musicales cn informations sonores. De plus, les structures du cortex pariétal impliquées dans cette misc en correspondance étaicnt situécs dans une région postérieure à cclle sous-tendant les opérations similaires dans le domaine verbal. Il semble donc qu'il existe une certaine indépendance des processus verbaux et musicaux à ce niveau, même si leurs substrats biologiques sont contigus.

3. La lecture et l'exécution au piano d'un morceau de musique, requérant des processus de déchiffrage, ont provoqué une activation intense du lobulc pariétal supéricur qui était demeuré silencicux lors de la lecture scule ou de l'exécution des gammes (figure 3). Cette activation doit donc être rcliée à la 

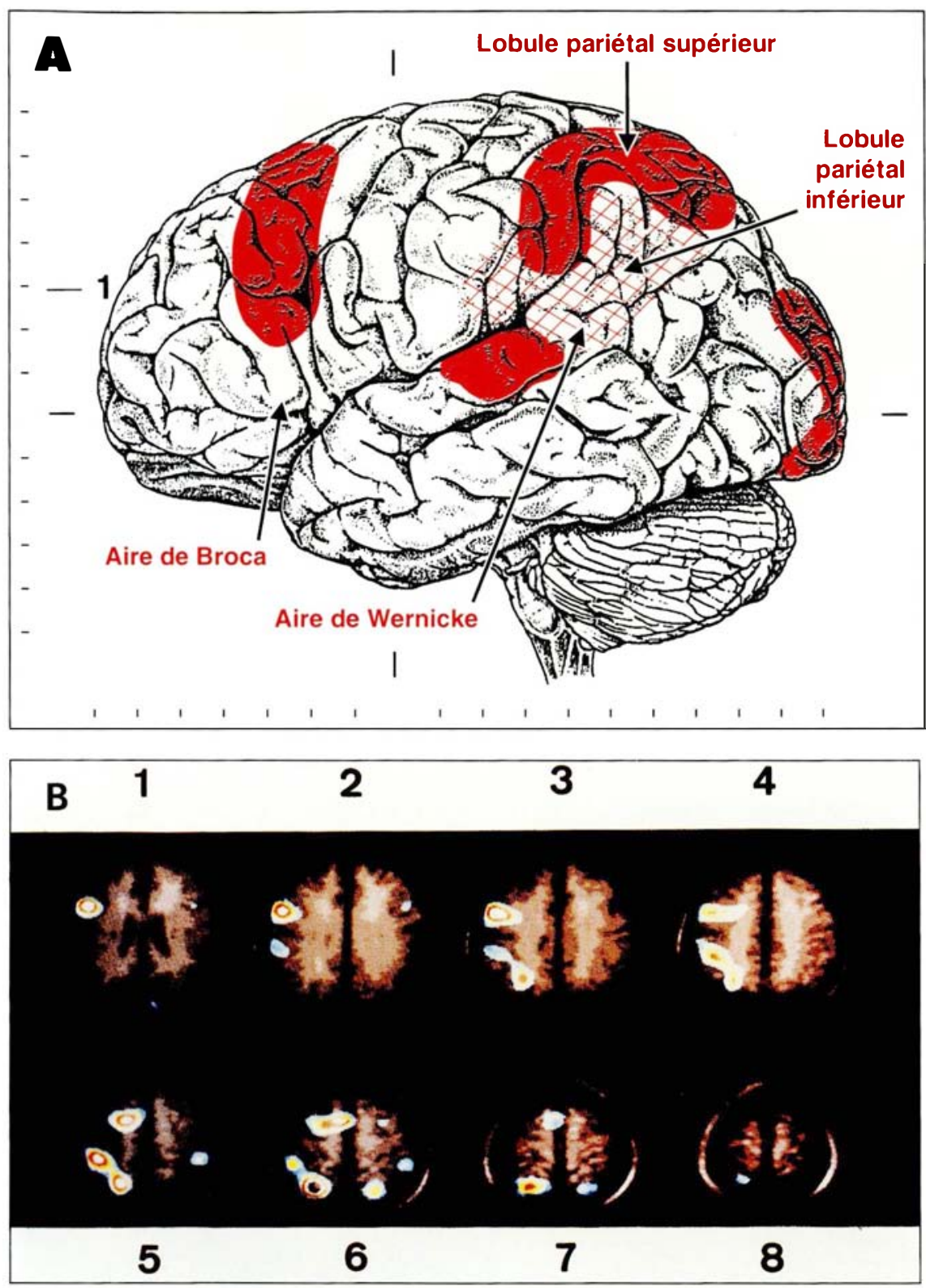

production d'informations spatiales, dérivées de la position des notes sur la portée ct visant à signaler au système motcur les distances devant séparcr les doigts pour jouer les notes sur le piano. D'autre part, unc seconde région spécifiquement activée durant cette tâche se situe juste au-dessus de l'aire de Broca dans le lobe frontal. L'aire de Broca joue un rôle critique dans l'organisation de la séquence des mouvements à effectuer dans la parole [30], et l'on peut donc penser que l'activation observée dans la région adjacente à l'aire de Broca indique un rôle similaire joué par cette $\mathrm{m} / \mathrm{s} \mathrm{n}^{\circ}$ I vol. 9, janvier 93 sujet joue de façon automatique des exercices maintes fois répétés et dont la séquence motrice est déjà apprise et établie.

A ces aires corticales spécifiquement activées lors du déchiffrage, il faut ajouter le cervelet dont la participation aux activités motrices est bien connue, et les aires temporales supérieures des deux hémisphè-
Figure 3. Illustration de certains résultats de l'étude tomographique par émission de positons. (A) représentation schématique du cerveau. La région hachurée correspond aux zones corticales dont la lésion provoque les déficits dont Maurice Ravel souffrait sur le plan des fonctions verbales, et elle comprend l'aire de Wernicke et le lobule pariétal inférieur (il est évidemment impossible de déterminer si toute cette zone était affectée chez Ravel, et il est probable, compte tenu de la sévérité des perturbations de l'écriture, de la lecture et du geste, que la partie supérieure de cette zone était la plus affectéel; les zones rouges correspondent aux régions spécifiquement activées durant la condition expérimentale de déchiffrage durant le TEP (puisque ces résultats sont obtenus par soustractions, beaucoup d'autres régions cérébrales non indiquées sont activées durant cette épreuve mais ne sont pas spécifiques au déchiffrage proprement dit). (B) Coupes horizontales du cerveau montrant les zones activées durant l'épreuve de déchiffrage, superposées sur les images des structures cérébrales des sujets obtenues par résonnance magnétique; ces coupes se situent dans la partie supérieure du cerveau, et le niveau de la coupe 1 est indiqué sur la figure de gauche; chaque coupe est séparée d'environ $6 \mathrm{~mm}$. Les zones activées correspondent à la différence d'activation entre la condition de déchiffrage et la condition de jeu et d'écoute des gammes, de sorte que l'activité purement motrice n'apparaît pas dans ces images. L'activation présente dans la partie antérieure des coupes 1 à 7 est située dans le cortex frontal et s'étend de la région située au-dessus de l'aire de Broca jusqu'au sommet du cortex dans une aire connue sous le nom $d^{\prime}$ " aire motrice supplémentaire ". L'activation présente dans la partie postérieure du cerveau (coupes 3 à 7) est située dans le cortex pariétal inférieur (coupes 3, 4, 5) et supérieur (coupes $5,6,71$. II est important de noter que dans l'épreuve de déchiffrage, les pianistes ne jouaient qu'avec la main droite; néanmoins, la coupe 6 montre que le cortex pariétal droit est activé durant la lecture musicale. 
res dont la contribution aux aspects réceptifs de la musique est établie depuis longtemps [35].

\section{Conclusions}

Les relations entre la musique et le cerveau sont évidemment complexes, mais l'on peut commencer à cerner certaines de leurs caractéristiques et à découvrir quelques-uns des principes qui les gouvernent.

(1) L'étude de ces relations montre à quel point la réalisation de fonctions musicales s'appuie sur un large réseau neuronal réparti sur les quatres lobes cérébraux dont chaque région possède une spécialisation fonctionnelle propre.

(2) Cette étude met aussi en relief l'importance, pour la mise en jeu de capacités musicales, de zones corticales servant à établir une correspondance entre des informations provenant de modalités différentes en effectuant unc traduction nécessaire à la communication et à l'intégration des diverses formes (visuelle, auditivc, motrice, mais aussi ćmotive) par lesquelles la musique peut être représentéc. Le lobe pariétal joue un rôle essentiel à cet égard, et les résultats de l'étude par TEP chez des pianistes professionnels apportent une validation des hypothèses émises sur la localisation des troubles neurologiques chez Ravel. Toutefois, sur la base des données physiologiques dérivées de la TEP, il est probable que la perturbation dont souffrait Ravel était plus étendue que ses seuls troubles verbaux nc le laisserait penser.

(3) Les résultats de l'étude par TEP confirme les dissociations des fonctions musicales suggérées par les déficits de Ravel. Cette convergence illustre les bénéfices à tirer d'approches expérimentales complémentaires qui permettent de mieux cerner le problème et de réduire les risques d'erreur.

(4) Les substrats neurobiologiques de la lecture et de l'exécution musicales sont distincts de ceux sous-jacents aux opérations correspondantes dans le domaine verbal. Néanmoins, le langage verbal et le langage musical, même s'ils portent sur des informations de nature différente au niveau visuel, auditif et sémantique, requiè- rent le même type de processus et de traduction d'informations entre les diverses modalités et font appel à des structures spécifiques situées dans les mêmes territoires corticaux. Cette organisation essentiellement parallèle mais adjacente de leurs substrats neuronaux rend donc probable, mais non nécessaire, la perturbation conjointe d'opérations verbales et musicales selon l'étendue et la localisation des lésions cérébrales.

On peut donc espérer, grâce aux nouvelles techniques d'exploration du cerveau et aux progrès des méthodes expérimentales neuro-cognitives, aboutir à une meilleure compréhension des relations entre la musique et les structures cérébrales. Il ne faut toutefois pas se leurrer sur la complexité d'une telle entreprise. Les connaissances acquises jusqu'à présent ne portent que sur les aspects les plus facilement contrôlables des fonctions musicales et celles-ci ne constituent qu'une fraction minime de ce qui fait l'essence même de la musique. Nous sommes en fait toujours ignorants des processus mentaux et cognitifs aussi bien que des substrats neuronaux par lesquels la musique suscite l'émotion et le plaisir esthétique, évoque des sentiments ou des tensions internes, et se trouve engendrée dans la "tête " d'un compositeur, autant d'aspects qui sont au cœur de l'expérience musicale et que la lésion dont Maurice Ravel fut victime avait épargnés

\section{RECHERCHE SUR LES ATAXIES HÉRÉDITAIRES}

Appel d'offre 1993 pour bourses et subventions de recherche

Afin de stimuler les recherches sur l'ataxie de Friedreich et les autres formes héréditaires d'ataxie, I'Association Française de l'ataxie de Friedreich (AFAF) attribuera, en 1993, des bourses de recherche d'un montant de 50000 à $150000 \mathrm{~F}$ pour un total de $300000 \mathrm{~F}$. Les domaines considérés comprendront les aspects fondamentaux (génétique, biochimie, neurobiologie) de ces maladies chez l'homme ou dans des modèles animaux, ainsi que la recherche clinique. Les formulaires à compléter sont disponibles auprès de : AFAF Mme G. Vachon, 15, rue de Bréau, 77240 Cesson, Tél. 60.63.20.42.

Date limite de dépót des demandes : 15 avril 1993

\section{Summary}

From music to brain, with Maurice Ravel

The understanding of the functional organisation of cerebral structures underlying receptive and expressive musical processes is confronted with a large variety of difficulties inherent in the artistic and subjective nature of the musical experience. Yet, clarifying the relationships between music and the brain is a legitimate goal of scientific research. One approach toward this goal is the study of musicians, such as Maurice Ravel, who suffered brain damage, and an analysis of their deficits make it possible to uncover some characteristics of these relationships, to identify the essential questions raised by these deficits, and to suggest some hypotheses about the neurofunctional anatomy of musical abilities. New developments in brain imaging such as positron emission tomography and magnetic resonance allow these hypotheses to be subjected to empirical verification. Results from such investigations indicate that the realization of musical abilities such as sight-reading and piano performance relies on a distributed neuronal network that encompasses the four cerebral lobes and the cerebellum, and it involves cerebral structures which are adjacent to, but distinct from, those underlying the realization of corresponding cognitive operations in the verbal domain.

\section{Remerciements}

Je remercie le professeur Jacques Racadot pour m'avoir communiqué le compte rendu de l'opération chirurgicale pratiquée sur Maurice Ravel et m'avoir autorisée à la publier. La préparation de cet article est rendue possible par l'octroi de subvention de la fondation EJLB, du conseil de la recherche médicale du Canada et du National Institute of Mental Health. 